\title{
Pre-SEgmentation fOR THE CoMputer Aided DIAGNOSIS SYSTEM
}

\author{
Myat Mon Kyaw \\ Faculty of Information and Communication Technology, \\ University of Technology (Yatanarpon Cybercity), Pyin Oo Lwin,Myanmar \\ monlay.mmkegmail.com
}

\begin{abstract}
This paper aims to introduce the method for detection and classification of an abnormality of the brain region into infarct, haemorrhage. Initially, the image is pre-processed to remove film artifact, noise using morphological operations. And the brain image is cropped to eliminate the extra parts. Before the abnormality region (infarct, haemorrhage) is segmented, the image is pre-segmented. In this stage, the image is subdivided into four quadrants using split and merge technique to find the region that has the possibility for inclusion of abnormal areas. Thus, the unnecessary regions are no need to search and segment. This paper proposes pre-processing and pre-segmentation steps for the detection of abnormal region in the brain image. Then, abnormalities can be segmented by grouping homogeneous regions according to the predefined criteria. The accuracy of the segmentation results can be assessed by the specialist.
\end{abstract}

\section{KEYWORDS}

CT, Medical imaging, Haemorrhage, Infarct

\section{INTRODUCTION}

Medical imaging refers to the techniques and processes used to create images of the human body for various clinical purposes. The analysis quality for medical image plays an important factor in the medical field [1]. The cases related to medical images are very complicated and important to diagnose correctly and treat disease in health care system [2].The success of medical image diagnosis depends on the quality of segmentation process. Medical images can be obtained from various imaging system such as MRI, CT etc. [1] [3]. Computed Tomography (CT) images are widely used in diagnosis of stroke due to wider availability, lower cost and sensitiveness to early stroke [4].The success for the diagnosis of medical image mainly depend on the accuracy of segmentation algorithm [5]. While various scanning techniques are almost always error free and quick in their operation, they require a competent Radiologist for the proper interpretation of the images generated. The algorithm and methodology will enable the easy and faultless identification of abnormalities present in the scanned region [4]. This paper organized as follows. Section 2 presents some related works. Section 3 discusses about the stroke. Section 4 presents some segmentation methods. Section 5 provides the stage of pre-processing. The pre-segmentation stage is explained in section 6 . The conclusion and further extension for the paper can be seen in section 7 . 


\section{RELATED WORKS}

T Kesavamurthy, Subha Raniand N Malmurugan proposed a Gabor filter technique for Computed Tomography image which is used for early diagnosis of human brain infarct [8]. Since Gabor filters are band-pass in nature, and infarcts lie in a particular frequency range, so choose only a subset of Gabor filter bank. The proposed image processing technique helps the radiologists to diagnose the early stage of brain infarct in CT image efficiently. It can only be used to detect brain infarct and the results were evaluated only on 53 patients. The condition for hemorrhage will not be passed by this filter and output image has no segmented portion. Although both types of stroke should be detected, most existing works on stroke detection mainly focus on hemorrhagic stroke detection [7]. Mayank Chawla, Saurabh Sharma, Jayanthi Sivaswamy, Kishore L.T presented a method for the detection and classification of an abnormality into acute infarct, chronic infarct and haemorrhage by the level of non-contrast CT images [4]. This system presents an algorithm based on symmetry to detect stroke affected region in a CT image [4]. The condition for symmetry will fail when the same type of stroke occurs symmetrically in both hemispheres of the brain [4]. This algorithm cannot handle such case. Segmentation is carried out based on a assumption that abnormalities are occurred in a single hemisphere with a healthy area on the other side. The dissimilarity between the left and right hemispheres of the brain is examined. ZHANG XiaoFeng, ZHANG CaiMing, TANG WenJing and WEI ZhenWen propose the improved Fuzzy C-Means algorithm based on the histogram of the given image, which can be denoted as HisFCM [9]. To classify the image into classes with the help of histogram, peaks can be denoted. The proposed method may fail in region of interest (ROI) retrieval in images because a segmentation method only using color information and statistical information provided by the given image. N. Mohd Saad, S.A.R. Abu-Bakar, Sobri Muda, M. Mokji, A. R. Abdullah presented fully automated region growing segmentation of brain lesion in diffusion-weighted MRI [10]. Split and Merge algorithm with quad tree structure is proposed. If four neighboring squares are found to be uniform, they are replaced (merged) by a single square composed of the four adjacent squares. The regions that are homogeneous according to certain criteria are merged to form Region of Interest (ROI). The resulted square may be greater or smaller than the real ROI. The segmented region may be same or not with the ROI.

\section{STROKE}

Stroke is a disease which affects vessels that supply blood to the brain and leading cause of severe disabilities [8]. When a brain blood vessel either burst or block, a stroke occurs [4]. Nerve cells in the affected brain area cannot be able to perform brain functions because of the lack of oxygen [4]. Stroke results in serious long term disability or death because the affected area of the brain cannot function. Two main types of stroke are: infarct and haemorrhage. Haemorrhage means blockage of a blood vessel supplying the brain due to lack of blood supply. Infarct means bleeding into or around the brain due to rupture of blood vessel. A brain CT image including haemorrhage region can be seen with a bright region inside it and has high contrast relative to its surrounds [4]. An ischemic (infarct) stroke appears as a dark region (hypo dense), with the contrast relative to its surround depending on the time elapsed since the stroke occurred [4] [6]. 

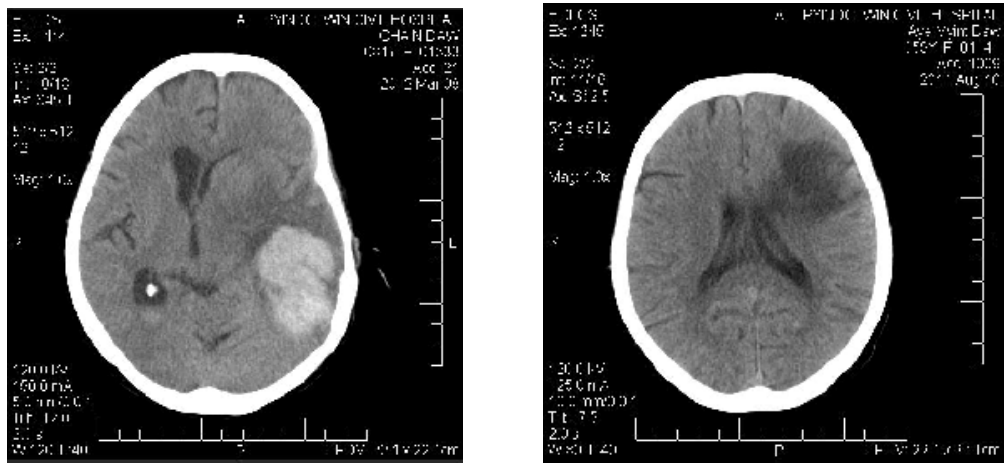

Figure 1. Brain CT images with infarct and haemorrh age region

\section{Segmentation Methods}

- Thresholding

Thresholding technique makes up of all the pixels with a certain threshold and establishes those pixels to the related areas [2]. Wrong threshold selection can cause the erroneous segmented results. These kinds of techniques are thus not reliable.

- Region growing techniques

It start with $\mathrm{n}$ pixels as seeds, each seed $\mathrm{i}$ is treated as a region, then $i$ region grows by adding its neighbour pixels with similar properties. A seed is required for each region to segment and decide appropriate threshold for homogeneity [12]. The Technique is not fully automatic.

- Supervised and Un-supervised Segmentation Methods

Supervised classification needs to know sufficient defined pixels to produce relative parameters for each class of Region of Interest (ROI) [13]. Some supervised segmentation methods are ML, k-NN, ANN. Un-supervised classification algorithm is efficient and less error sensitive, such as k-means, hierarchical clustering, self organizing map (SOM), normalized cut algorithm, fuzzy cmeans and so on.

\section{PRE-PROCESSING}

In medical image processing, pre-processing is very important so that segmentation and feature extraction algorithms work correctly.

\subsection{Noise Removal}

Pre-processing stage is performed for film artifact and noise removal and for intensity enhancement. The CT image consists of film artifact or labels on the CT such as patient name, age and marks. Film artifacts and unwanted portions are removed by applying morphological operations, erosion and dilation. 

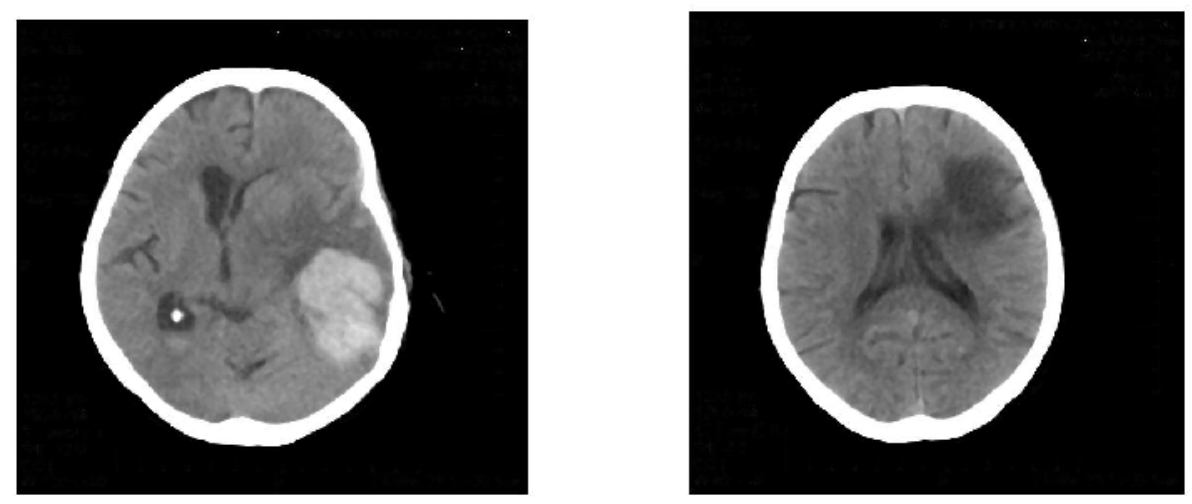

Figure 2. Resulted images from pre-processing
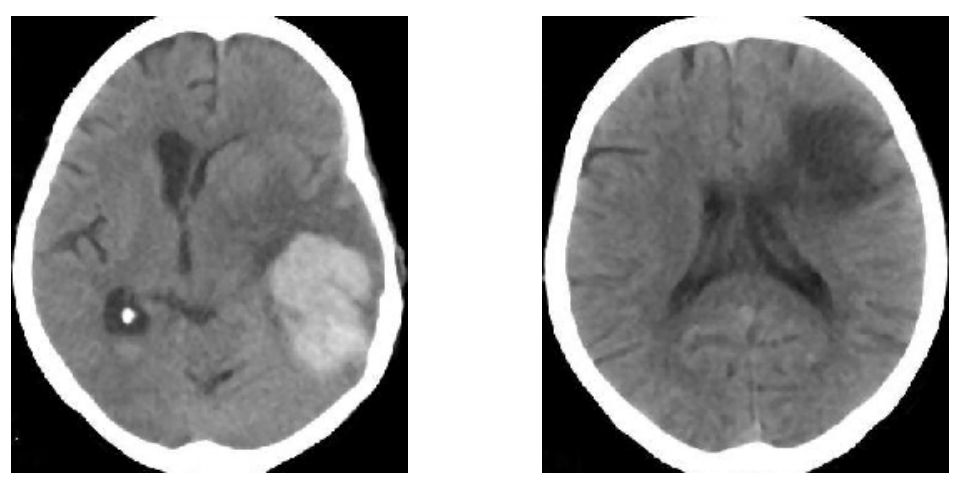

Figure 3. Resulted images after cropping unwanted parts

\section{Pre-Segmentation}

In the pre-segmentation stage, the image from pre-processed stage is broken into four quadrants (regions). And one quadrant (region) that has the possibility for inclusion of abnormal areas is divided into four sub regions using the statistical feature before clustering is carried out. The unnecessary regions are no need to search and cluster. So it can conclude that the region' mean value is higher than the other regions, it may contain haemorrhage area. The region that contains infarct area may have low mean value. Statistical features, mean and standard deviation, are used to distinguish the normal and abnormal regions. Mean and standard deviation are the statistical features that are frequently used. Mean is the division of sum of all pixel values by the number of pixel in an area [14]. For a grey scale image this is equal to the average brightness or intensity [14]. Standard deviation is the variation from its mean. They can be defined by Eq. (1) and Eq. (2) respectively.

$$
\begin{aligned}
& \mu=\frac{1}{N} \sum_{i=1}^{N-1} P(i) \\
& \sigma=\sqrt{\frac{1}{N} \sum_{i=0}^{N-1}(P(i)-\mu)^{2}}
\end{aligned}
$$


$i=$ random variable representing the $\mathrm{N}$ intensity levels of the image, $P(i)=$ histogram of $i$.

The proposed steps for pre-segmentation are:

Step 1: The image is divided into 4 quadrants.

Step 2: Compute the histogram and pixel values for each quadrant.

Step 3: Compare each with the predefined criteria (histogram, pixel value).

Step 4: Only the abnormality contained region is fed into segmentation process.

\subsection{Experimental results}

In pre-segmentation stage, only the region that contains abnormal part is selected for segmentation stage. Figure 2 shows the histogram that is mostly found model in the haemorrhage areas. At the pre-segmentation stage, the region that may contain abnormal area is selected among the regions according to its histogram and pixel value. Figure 3 illustrates the four quadrants and Figure 4 demonstrates their related histograms.

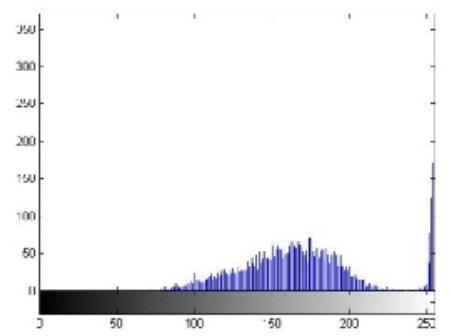

Figure 4. Histogram of hemorrhage region

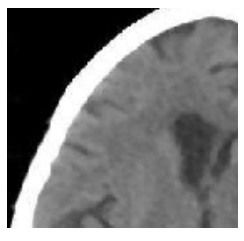

Figure 5(a). region 1

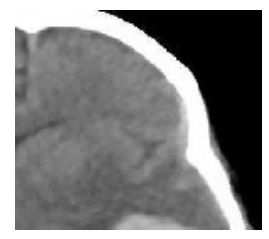

(b). region 2

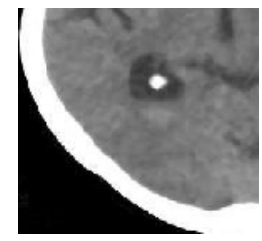

(c). region 3

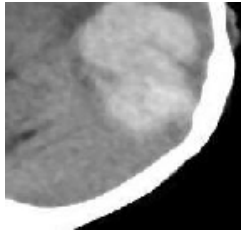

(d). region 4

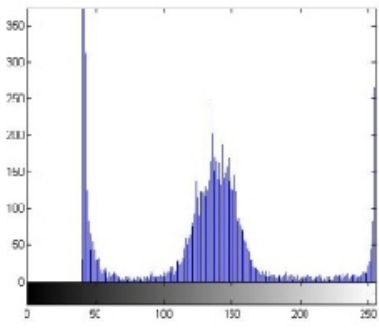

Figure 6(a). Histogram of region 1

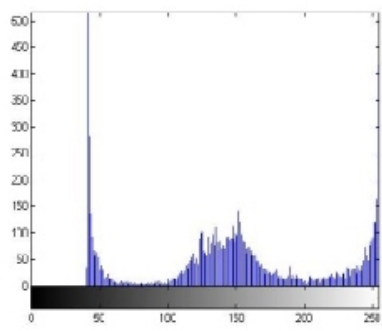

(b). Histogram of region 2 


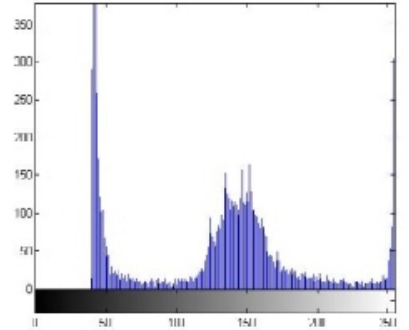

(c). Histogram of region 3

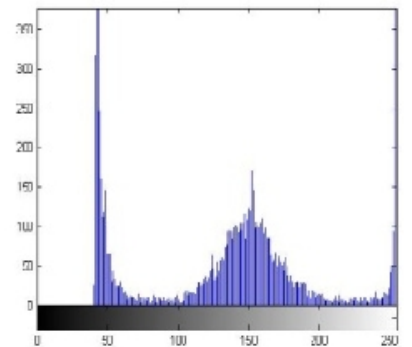

(d). Histogram of region 4

As shown in figures, only region (2) has the possibility for inclusion of abnormal area. Because only region (2) has the pixel value under 100 that are generally found in abnormal region and hence has the most similar histogram model. In reality, haemorrhage region is located in region (2). So region (2) is fed into the further detail segmentation step.

\section{CONCLUSION AND FURTHER EXTENSION}

The proposed system is to produce precise segmentation of brain images using intensity information along with neighbourhood relationships and histograms. The proposed presegmentation steps are carried out in this paper. This pre-segmentation process makes the segmentation process faster than the ordinary process. The final segmentation results tested upon the regions resulted from pre-segmentation and the accuracy rates of the proposed system will be described in the further works. The brain CT images are segmented into their separate tissue types and will result in abnormal tissue being easier to identify. And the system assists the radiologists to identify the haemorrhage and infarct regions in the human brain and to arrive at a decision faster and accurate. Radiologists use the results from the computer as a second opinion to make final decisions. The accuracy for the proposed system will be assessed by the knowledge of the technicians and specialists in radiology. The system can be extended to detect other brain abnormalities - tumour, abscess and lesion etc. The adaptive algorithms can be applied in other medical images such as lung, liver, and bone to detect their related diseases such as cancer, tuberculosis.

\section{REFERENCES}

[1] A. Padma and Dr.R. Sukanesh, "Automatic Diagnosis of Abnormal Tumor Region from Brain Computed Tomography Images Using Wavelet Based Statistical Texture Features", International Journal of Computer Science, Engineering and Information Technology (IJCSEIT), Vol.1, No.3, August 2011.

[2] M. Rastgarpour, J. Shanbehzadeh, IAENG, " Application of AI Techniques in Medical Image segmentation and Novel Categorization of Available Methods and Tools" , Proceedings of the International MultiConference of Engineers and Computer Scientists, Volume ,2011,IMECS 2011, March 16-18, Hong Kong, 2011.

[3] A.Padma \& Dr.R.Sukanesh, "A Wavelet Based Automatic Segmentation of Brain Tumor in CT Images Using Optimal Statistical Texture Features", International Journal of Image Processing (IJIP), Volume (5) : Issue (5) : 2011.

[4] Mayank Chawla, Saurabh Sharma, Jayanthi Sivaswamy, Kishore L.T, "A method for automatic detection and classification of stroke from brain CT images".

[5] Iraky khalifa , Aliaa Youssif , Howida Youssry ," Tissue segmentation Techniques of brain MR Images: A Review", International Conference on Intelligent Computational Systems (ICICS'2012), Dubai, Jan. 7-8, 2012. 
[6] T Kesavamurthy, S SubhaRani, "Pattern Classification using imaging techniques for Infarct and Hemorrhage Identification in the Human Brain", Calicut Medical Journal 2006; 4(3):e1.

[7] T. L. Tan, K. S. Sim, C. K. Tan1, and A. K.Chong, "CT Image Enhancement by Colorization for Brain Infarct Detection", Malaysia.

[8] T Kesavamurthy, Subha Rani and N Malmurugan, "EARLY DIAGNOSIS OF ACUTE BRAIN INFARCT USING GABOR FILTER TECHNIQUE FOR COMPUTED TOMOGRAPHY IMAGES”, Biomedical Soft Computing and Human Sciences, Vol.14, No.1, pp.11-16 (2009).

[9] ZHANG XiaoFeng, ZHANG CaiMing, TANG WenJing and WEI ZhenWen, "Medical image segmentation using improved FCM", Vol. 55 No. 5: 1052-1061, May 2012.

[10] N. Mohd Saad, S.A.R. Abu-Bakar, Sobri Muda, M. Mokji, A.R. Abdullah, “Fully Automated Region Growing Segmentation of Brain Lesion in Diffusion-weighted MRI”, IAENG International journal of computer science, 39;2, IJCS_39_2_03,2012.

[11] Sonali Patil, Dr. V. R. Udupi, "Preprocessing To Be Considered For MR and CT Images Containing Tumors", IOSR Journal of Electrical and Electronics Engineering (IOSRJEEE), Volume 1, Issue 4, PP 54-57, July-Aug. 2012.

[12] Neelum Noreen, Khizar Hayat and Sajjad A. Madani, " MRI Segmentation through Wavelets and Fuzzy C-Means", World Applied Sciences Journal 13 (Special Issue of Applied Math): 34-39,ISSN 1818-4952, (C IDOSI Publications, 2011.

[13] M. Masroor Ahmed \& Dzulkifli Bin Mohammad," Segmentation of Brain MR Images for Tumor Extraction by Combining Kmeans Clustering and Perona-Malik Anisotropic Diffusion Model", International Journal of Image Processing, Volume (2) : Issue(1).

[14] Torsten Seemann ," Digital Image Processing using Local Segmentation book", Monash University Australia, April 2012.

\section{AUTHOR}

Myat Mon Kyaw was born in Mandalay Division, Myanmar, in 1987. The da te of birth is August 20, 1987. She received the B.C.Sc (Bachelor of Computer Science) and M.C.Sc (Master of Computer Science) degrees in computer science from University of Computer Studies (Mandalay), Myanmar in 2006 and 2009, respectively. She is currently a candidate for the Ph.D degree in information technology at the University of Technology (Yatanarpon Cybercity), Myanmar.

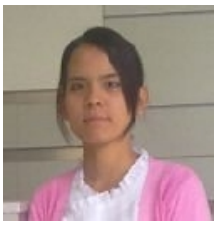

From 2010 to now, she is working as a tutor at the faculty of Information and Communication Technology, University of Technology (Yatanarpon Cybercity), Myanmar. Her research interests include digital image processing, computer aided diagnosis system. 